\title{
Über großknotige Lebertuberkulose kombiniert mit Leberzirrhose unter dem Bilde einer Leber- vergrößerung.
}

\author{
Von \\ Dr. W. Culp, Barmen. \\ (Aus dem pathologischen Institut des städtischen Krankenhauses in Mainz. \\ [Leiter: Gg. B. Gruber].)
}

Die großknotige Lebertuberkulose gehört mit zu den seltensten Erkrankungen. Im Jahre 1912 konnte Lotheissen unter Heranziehen aller nur möglichen Fälle nur 34 Beobachtungen zusammenstellen. Seitdem sind besonders von seiten der Chirurgen wohl einige neue veröffentlicht worden, w'esentlich Neues über die pathologische Anatomie ist aber dabei nicht gebracht worden. Ich möchte nun im folgenden Falle weniger die Kasuistik vergrößern, wie auf einen Punkt hinweisen, der mir widerspruchsvoll erscheint und der Aufklärung dringend bedarf, auf den Infektionsweg. Da sich in unserem Falle außerdem eine ganz groteske Bindegewebsvermehrung und Lebervergrößerung fand, wie sie bisher nur von Isaak beschrieben ist, so wird auch die Frage, ob wir es mit einer tuberkulösen Leberzirrhose zu tun haben. zu erörtern sein.

G. K., 53 Jahre, Privatier. Früher Besitzer einer Weinwirtschaft. War im Laufe des letzten Jahres verschiedentlich kürzere Zeit mit den Beschwerden einer Insufficientia cordis in Behandlung. Es wurde außer einer geringen Verbreiterung des Herzens eine deutliche Vergrößerung der Leber und der Milz festgestellt. Beim letzten Krankenhausaufenthalt im Juli 1919 Aszites mäßigen Grades. Kam am 31 . August 1919 mit den Zeichen einer hochgradigen Herzinsuffizienz wieder. Die klinische Untersuchung ergab außer den vorher beschriebenen Befunden noch eine sehr starke Flüssigkeitsansammlung in beiden Pleurahöhlen. Auch diesmal wurde, wie schon früher, die Wassermannsche Reaktion angestellt, sie war negativ. Der Tod trat am 2. September unter den Zeichen zunehmender Herzschwäche ein.

Obduktion 6 Stunden p. $\mathrm{m}$. Leiche eines $180 \mathrm{~cm}$ großen Mannes in sehr gutem Ernährungszustand. Totenstarre an allen Gelenken deutlich, reichlich Totenflecke an den abhängigen Partien des Körpers. Pupillen gleichweit, Augen gerade gerichtet.

Fettpolster des Bauches sowie Bauchdeckenmuskulatur gut entwickelt. In der freien Bauchhöhle, deren peritonealer Überzug glatt und glänzend ist, etwa $2500 \mathrm{ccm}$ einer trüb serösen Flüssigkeit. Das fettreiche Netz ist nach unten ausgezogen und läuft in einem derben Strang zum linken Leistenkanal, wo es verwachsen ist. Der Leistenring ist weit.

Zwerchfellstand beiderseits in Höhe der 6. Rippe. Beide Lungen sind stark komprimiert, sie sind nicht mit dem Brustfell verwachsen. In der linken Pleurahöhle $1000 \mathrm{ccm}$ einer etwas hämorrhagischen Flüssigkeit, rechts etwa $3000 \mathrm{ccm}$ eines trüb-serösen Ergusser. Der Herzbeutel enthält geringe Mengen Transsudat.

Beiträge zur Klinik der Tuberkulose. Bd. 46 . 
Auf den Pleuren beider Lungen erkennt man zahlreiche Auflagerungen in der Größe eines Hirsekorns. Die Sehnittflächen zeigen außer einer Kompression des Gewebes keine deutlichen Veränderungen. Jegliche Einlagerungen von Knötchen fehlen. Die Hilusdrüsen sind klein und mit Kohlestaub imprägniert. Die Schleimhaut der Bronchien ist gerötet, sie sind zum Teil mit Schleim angefüllt.

An den Halsorganen ist außer einer Rötung der Trachealschleimhaut ein krankhafter Befund nicht $\mathrm{zu}$ erheben.

Auch die Obduktion des Herzens läBt außer einer geringen Dilatation und Blässe der Muskulatur keine Besonderheiten auffinden. Aorta und Koronargefäße zeigen nur eine unbedeutende Fleckung.

Die Milz ist außerordentiich stark vergrößert (26:18:6). Gewicht $1300 \mathrm{~g}$. Ihre Kapsel ist von einer nahezu $75 \mathrm{~mm}$ dicken Bindegewebsschicht bedeckt; die zahlreiche höckerige Auflagerungen zeigt. "Sie ist sehr derb. Anf dem Sehnitt, der braunrot verfärbt ist, sind ïberall Knoten von weißlicher Farbe in Hirsekorn- bis Bohnengröße zu finden.

Die Leber ist mit ihrer Umgebung stark verwachsen. Sie ist sehr groß $(32: 27: 22 \mathrm{~cm})$. Gewicht $3450 \mathrm{~g}$. Ihre ganze Oberfläche ist von einer derben, dichten, bindegewebigen Haut überzogen. Sie ist sehr hart. Auf dem Schnitt sieht man in einem vielfach ockergelben bis bräunlichen, meist gut gezeichneten Parenchym zahlreiche weiße, gut umschriebene Knoten, die einen Durchmesser bis zu $11 / 2 \mathrm{~cm}$ erreichen und in dieser Größe meist unter der Oberfläche liegen. Kleinere Knoten sind in Unmengen über die ganze Leber verteilt. Eine Bevorzugung irgendeines bestimmten Teiles läßt sich nicht wahrnehmen. Zwischen dem Lebergewebe lassen sich mit bloßem Auge Züge von Bindegewebe erkennen. An der Leberpforte ist ein etwa pflaumengroßer derber Knoten, der sich in einzelne bis bohnengroße, zum Teil verkäste Lymphdrüsen auflösen läßt. Die Gallenblase ist unverändert.

Die Nieren sind ebenfalls stark vergrößert. Sie lassen sich leicht entkapseln. Ihre Oberfläche ist glatt. Die Schnittfläche ist tief dunkelrot, der Übergang der Rinde ins Mark ist nicht deutlich.

An den ableitenden Harnwegen und am Mastdarm keine Veränderungen. Ebenso scheinen die übrigen Organe des Bauches, von einer erheblichen Hyperämie abgesehen, nicht verändert.

Die Wassermannsche Reaktion, die mit dem. Leichenblut vorgenommen wurde, war negativ.

Da makroskopisch eine Tuberkulose in Frage kam, so wurden zur Sicherung der Diagnose kleine Teile der Leber mit den in Frage stehenden Knoten steril entnommen und in physiologischer Kochsalzlösung zerkleinert. Mit dieser Flüssigkeit wurden zwei Meersch getötet. Beide zeigten die gleichen Veränderungen. Erbsen- bis bohnengroße Drüsen unter der Bauchhaut und weiße hirsekorn- und erbsengroße Knoten in Milz und Leber, in deven mikroskopisch die Tuberkulose sichergestellt und zahlreiche Bazillen nach der Ziehlschen Methode nachgewiesen wurden. Fine besondere bindegewebige Reaktion in der Leber der Versuchstiere wurde vermißt.

Pathologisch-anatomisehe Diagnose:

Großknotige Tuberkulose der Leber und der Milz mit enormer. VergröBerung beider Organe. Perihepatitis und Perisplenitis. Tuberkulose der Leberhilusdrüsen. Aszites. Staungsnieren. Atelektase beider Lungen. Pleuritis tuberculosa. Leistenhernie links.

Die histologische Untersuehung ergab in der Leber folgenden Befund:

Die makroskopisch erkennbaren Knoton erwiesen sich meist als strukturlose käsige Masse, die durch einen bindegewebigen Wall gegen das umgebende Gewebe abgeschlossen waren. Dieser Wall bestand aus einem sehr kernarmen, in deutlichen zirkulären Zügen angeordneten hyalinen Bindegewebe. Erst in seinen an das Lebergewebe angrenzenden Teilen wurde es kernreicher. Hier ist es auch ab und zu von farblosen Blutelementen durchsetzt. Gefäßquerschnitte werden hier ebenfalls in größerer Zahl deutlich. In den gleichen Präparaten finden wir typische Tuberkel von wechselnder Größe aus Epitheloidzellen mit zahlreichen Langhansschen Riesenzellen. Auch diese Tuberkel sind zum größten Teil bindegewebig abgegrenzt. Schließlich sind miliare Tuberkel ohne deutlichen Abschlu[3 gegen die Umgebung in großer Zahl vorhanden. Alle diese Knötchen liegen in mächtigen Bindegewebsziugen, dio vollkommen unregelmäßjg das ganze Organ durchziehen. Begleitet 
sind diese Züge von enormen Mengen von weißen Blutelementen. Eine Abhängigkeit des Bindegewebes von den Tuberkeln ist nicht zu erkennen. Ebenso wird eine bestimmte Anordnung durchaus vermißt, es verläuft an einigen Stellen inter-an anderen intráazinös, um an wieder anderen dann in keiner Weise auf die Struktur der Azini irgendwelche Rücksicht zu nehmen. Größere und kleinere Bezirke von Leberzellen sind abgetrennt, oft liegen nur ein oder zwei Leberzellen in diese Züge eingelagert. Mit Hilfe der van Gieson. färbung läßt sich außerdem feststellen, daß auch das intralobuläre Gewebe an dem Prozeß beteiligt ist, da diese Fasern, die sonst nur mit Hilfe der Silberimprägnation darzustellen sind, hier auch in leuchtendroter Farbe hervortreten. Besonders stark ist auch die Bindegewebsvermehrung der Wandungen der Zentralvenen. Gallengänge lassen sich in allen Präparaten in großer Zahl nachweisen. Sie sind relativ groß und vielfach geschlängelt. Neugebildete Leberbezirke sind mit Sicherheit nicht zu erkennen. Die Färbung auf Tuberkelbazillen zeigte zahlreiche Exemplare in den mi.iaren Knötchen.

Die Milz zeigte eine erhebliche bindegewebige Verdickung ihrer Kapsel. Auch das trabekuläre Bindegewebe war vermehrt. Tuberkel der verschiedensten Größe konnten auch hier histologisch nachgewiesen werden.

Fassen wir die Befunde des vorliegenden Falles kurz zusammen, so haben wir bei einem Mann, der ein halbes Jahr vor seinem Tode mit den Zeichen einer Herzinsuffizienz. und Beschwerden von seiten der Leber erkrankt, eine großknotige Lebertuberkulose mit LebervergröBerung bedingt durch eine übermäßige Bindegewebsentwicklung.

Wenden wir uns zunächst der g roßknotigen Tuberkulose zu, so müssen wir wiederum feststellen, daß es sich um einen sehr seltenen Befund handelt. Dies ist um so auffallender, als doch schon lange bekannt ist, daß die Leber bei einem sehr großen Prozentsatz aller Phthisen miterkrankt. Meist handelt es sich um miliare Knötchen, die nach der Ansicht der Mehrzahl der Autoren erst gegen Ende des Lebens entstehen, wenn die Widerstandskraft des Körpers auf ein Minimum reduziert ist. Gewöhnlich werden die Tuberkelbazillen, die ja auf dem Blutweg in großen Mengen in die Leber gelangen, von den Leberzellen vernichtet, wobei die antibakterielle Kraft der Galle eine besonders große Rolle spielen soll.

Eine Erkrankung, bei der besonders viele Tuberkelbazillen der Leber zugeführt werden, ist nach den Untersuchungen von Strauß und den Erfahrungen von B. Fischer die Darmtuberkulose, bei der im Pfortaderblut mit großer Regelmäßigkeit Bazillen nachgewiesen werden können. Man müßte daher annehmen, daß bei dieser Erkrankung besonders häufig großknotige Lebertuberkulosen gefunden würden. Das ist aber keineswegs der Fall. Im Gegenteil wird besonders in der letzten Zeit darauf hingewiesen, daß größero Knoten in der Leber oft ohne sonstige tuberkulöse Erkrankungen vorkommen und daher die operative Entfernung solcher Knoten eine recht gute Prognose gibt. Ja, manche Autoren haben gemeint, daß die von ihnen gefundenen tuberkulösen Knoten der Leber als primäre Tuberkulose anzusprechen seien. Auch Isaak, der einen Fall von tuberkulöser Leberzirrhose veröffentlichte, welche mit dem vorliegenden manche Ähnlichkeit hat, sieht ihn als primäre Lebertuberkulose an.

Wir sehen also auf der einen Seite Erkrankungen, bei denen massenhaft Tuberkelbazillen der Leber zugeführt werden und bei denen es zu keiner oder höchstens zu einer miliaren Aussaat kommt und auf der anderen Seite finden wir keine oder nur sehr geringfügige tuberkulöse Herde im Körper; trotzdem entsteht auf dem an sich sehr ungünstigen Nährboden eine großknotige Leber- 
tuberkulose. Dieser Widerspruch wurde meiner Meinung nach bisher nicht genügend beachtet. Er scheint mir nur auf eine Weise zu erklären. Wir müssen annehmen, daß in derartigen Fällen der Leber die Tuberkelbazillen nicht auf dem Blutweg, sondern auf dem Lymphweg zukommen. Dadurch allein kann es zu der notwendigen Anhäufung von Tuberkelbazillen kommen, die imstande ist, in einem so ungünstigen Nährmedium, wie es die Leber angeblich darstellt, eine Erkrankung von so großem Umfang hervorzurufen. Mit den Bazillenmengen, die auf dem Blutweg in rascher Verteilung in die Leber gebracht werden können, wird das Organ im allgemeinen verhältnismäßig leicht fertig werden, es dürften hierbei auch die einzelnen Ablagerungsstellen (Embolieorte) nicht so massig infiziert werden.

Um die Berechtigung dieser Anschauung zu erweisen und zu zeigen, daß der Lymphweg überhaupt möglich ist, müssen wir etwas weiter ausholen. Es war in neuerer Zeit Tendeloo, der wieder darauf hingewiesen hat, daß wir viel mehr wie bisher an eine lymphogene Verschleppung von Krankheitserregern zu denken haben. Er steht außerdem auf dem Standpunkt, daß die Möglichkeit einer rückläufigen Bewegung der Lymphe sehr in Betracht zu ziehen ist, daß also auf dem Lymphwege eine Verbreitung von Keimen aus der Brusthöhle über die subphrenisch gelegenen Lymphdrüsen hinaus tiefer in die Bauchhōhle hinein unter gewissen Umständen durchaus annehmbar sei. Er weist auf die Fälle hin, wo Staubpigment in den Lymphgefäßen des Zwerchfells und in den paraaortischen Drüsen gefunden wurde. Den Untersuchungen Kuettners verdanken wir sodann die Kenntnis der Wege, auf denen solche Verschleppungen zustande kommen können. Ob nun tatsächlich die Kohleteilchen retrograd auf dem Lymphweg verschleppt werden, ist noch nicht mit Bestimmtheit entschieden, eine Reihe von Autoren (Beitzke, Askanazy u. a.) hielten auch da den Blutweg für den gewöhnlichen, während Lubarsch die Fälle von isolierter periportaler Lymphdrüsenanthrakose bei Freisein der Leber nur durch den retrograden Lymphtransport entstanden glaubt. Auch Franke kommt zu dem SchluB, daß ,die retroperitone:sen Drüsen an der Arteria coeliaca und von da aus abwärts bis nahezu an die Bifurkation der Aorta, ferner die Drïsen bis zum Hilus der Milz, Leber und Nieren in direkter Verbindung mit den Lungenlymphdrüsen stehen und Staub und Bakterien auf direktem Wege dorthin verschleppt werden können". Wie auch die Verhältnisse bei einer Verschleppung von Staubteilchen liegen mögen, die rückwärtige Ausbreitung von Infektionserregern läßt sich nicht bestreiten, wenn es gelingt, die infizierten Lymphdrüsen Schritt für Schritt zu verfolgen, ja man bezeichnet jetzt geradezu die an der kleinen Magenkurvatur gelegenen Lymphdrüsen als ständige Ablagerungsgelegenheiten von Lymphpartikelchen aus dem Lungengebiet. Die Lungen sind diesen Drüsen tributär (Beitzke). Was nun die sehr oft konstatierbare gleichzeitige Infektion von Milz und Leber angeht, so meint Ziegler, daß die Verschleppung von Keimen durch längs der Milzvene verlaufende Lymphbahnen das gewöhnliche sein soll.

Vor kurzem ist es nun Straub gelungen, auf Grund ausgedehnter Versuche für Kaninchen und Meerschweinchen die Richtigkeit der oben erwähnten Annahmen zu erweisen. Er konnte bei diesen Tieren unter Umgehung des MagenDarmkanals durch Injektion von tuberkulösem Material in das große Netz 
und das Mesenterium einer Dünndarmschlinge Leber- und Milztuberkulosen erzeugen. Er betont, daß diese Organe nur erkranken, wenn die zugehörigen Lymphdrüsen in der Gegend des Pankreaskopfes infiziert sind. Er konnte weiter mit Sicherheit verbindende Lymphwege zwischen Milz und Leber feststellen, so daß das eine Organ ohne Schwierigkeit durch das andere infiziert werden kann. Schließlich gelang ihm der Nachweis von Lymphverbindungen zwischen Brust- und Bauchhöhle sowie der einer Infektion der Bauchorgane von den Lymphdrüsen der Brust aus.

Wieweit stimmen nun diese Versuche mit den Erfahrungen auf dem Obduktionstisch überein? Die Tuberkulose der Erwachsenen kann uns darüber meist keine Auṣkunft geben, denn bei ihnen scheint die lymphogene Verbreitung nicht das Gewöhnliche zu sein. Bei den Erwachsenen steht ja überhaupt der lymphatische Apparat nicht mehr so im Vordergrund wie beim kleinen Kind. Man wird sich daher wohl auf die Kindertuberkulosen stïtzen müissen. Außerdem pflegen, wie unsere Erfahrung lehrt, gewisse wenig durchseuchte Negerrassen in erwachsenem Alter in der gleichen Weise zu erkranken, wie es die europäischen Kinder tun, nur sind die Formen grotesker, da ihre Widerstandskraft vielleicht doch eine größere ist. Wir waren in letzter Zeit in der Lage, eine größere Anzahl solcher Neger-Phthisiker zu untersuchen und an ihnen die Eigenart des uns hier interessierenden pathologisch-anatomischen Bildes zu studieren.

Bei ihnen finden wir, wie Gg. B. Gruber an anderem Ort ausgeführt hat, mit großer Regelmäßigkeit eine außerordentlich ausgedehnte Tuberkulose der Tracheal- und Bronchialdrüsen. Lymphdrüsenpakete von Mannsfaustgröße sind keine Seltenheit. In fast allen Fällen sind die Achseldrüsen erkrankt. Dann findet man die Ausbreitung durch das Zwerchfell hindurch. Wir haben fast in keinem der einschlägigen Neger-Fälle eine Tuberkulose der retroperitoneal gelegenen, an der Aorta entlang laufenden Drüsen vermißt. Ebenso waren die im kleinen Netz gelegenen fast stets ergriffen. Auch an der Milz- und Leberpforte konnten sie festgestellt werden. Bei einem Fall gelang es mir, der Milzvené entlang eine vollkommene Kette von tuberkulös erkrankten Drüsen von der Milz bis zur Leber zu finden. Leberund Milztuberkulosen wurden auch bei makroskopischer Betrachtung selten vermißt. Herde in den beiden. Organen, die eine Größe einer Kirsche hatten, konnten oftmals konstatiert werden. Bei diesen Formen waren die Driisen an der Leberpforte, soweit darauf geachtet wurde, stets ergriffen, aber auch wenn sich in der Leber nur miliare Herde fanden, sah man gelegentlich tuberkulöse Drüsen an der Leberpforte.

Tuberkulöse Pleuritiden und Peritonitiden waren recht häufig. Seltener. konnten Nierentuberkulosen und Erkrankungen der Generationsorgane festgestellt werden. Zu einer Miliartuberkulose kam es erst gegen Ende der meist 4-6 Monate dauernden Erkrankung, wenn der Tod nicht durch Wirkung großer Exsudate oder käsige Pneumonie erfolgte. Wir sehen ałso hier in der Hauptsache den Lymphweg zur Ausbreitung der Erkrankung benutzt und finden großknotige Leber- und Milztuberkulosen, die bei den erwachsenen Europäern zu den Seltenheiten gehören, als etwas ganz Alltägliches. Es wäre uns ein Leichtes gewesen, in der kurzen Zeit eines Jahres aus dem Neger- 
material fast die gleiche Anzahl von solchen Fällen zusammenzustellen, die Lotheissen aus der gesamten Literatur gesammelt hat.

Ich glaube daher, auch für die großknotigen Abdominaltuberkulosen der Europäer eine vorwiegend lymphogene Infektion annehmen zu müssen: Bei meinem Fall konnte ich auch die Tuberkulose der Leberhilusdrüsen nachweisen, was für sich allein natürlich nie beweisend wäre, da die Drüsen bei einer so ausgedehnten Erkrankung des Organs auch von dorther infiziert sein könnten, was aber angesichts aller bisher angeführten Tatsachen wohl bezweifelt werden kann. Ich betone aber ausdrücklich, daß ich mit der Feststellung der lymphogenen Infektion bei der großknotigen Tuberkulose der Leber meines Falles nur den Weg von den Drüsen der Leberpforte zu dem Organ selbst bezeichnet haben möchte. Sicheres über die Art, wie die Erkrankung der Drüsen zustande kam, kann ich nicht sagen. Ob die Infektion von der Brust her erfolgte, oder ob eine tuberkulöse Affektion im Bauchraum vorausging oder ob sich ein älterer Herd nicht auffinden läßt, das steht naturgemäß in jedem einzelnen Fall zur Frage.

Wenden wir uns nun der zweiten Leberveränderung zu, der Vergrößerung durch die übermäßige Bindegewebsentwicklung, so werden wir in Anbetracht der bestehenden Tuberkulose ohne weiteres darauf geführt, hier an eine tuberkulöse Leberzirrhose zu denken. Wie steht es mit dieser Erkrankung?

Von Jahr zu Jahr mehren sich die Arbeiten, in denen dargetan wird, daß tuberkulöse Leberzirrhosen vorkommen und trotzdem scheint sie sich in den Kreisen der Pathologen wie auch insbesondere der Kliniker keine rechte Geltung verschaffen zu können. Das hat in erster Linie zwei Gründe. Die Arbeiten der Pathologen sind oft dem Kliniker nicht so gut zugänglich, wie es wohl wünschenswert wäre, und dann -wird manchmal in dem . Wort ,tuberkulöse Zirrhose" ein Angriff auf die zirrhosierende Wirkung des Alkohols gesehen. Aber es ist vielleicht noch eine Ursache, die die Ablehnung begründet, und die wiegt schwerer. Es scheint mir hier das „Primum definiere“" außer acht gelassen zu sein. Wir müssen diese Selbstverständlichkeit, wie sie E. Albrecht nennt, erfüllen, sonst nehmen die Meinungsverschiedenheiten nie ein Ende.

Gehen wir von der Definition der Leberzirrhose aus, wie sie uns K retz gegeben hat und wie sie anscheinend noch heute allgemeine Gültigkeit hat. Sie ist nach ihm ein chronischer, herdweise rekrudeszierender Degenerationsprozeß mit eingeschobener Regeneration von Leberparenchym. Diese Anschauung setzt die Ackermannsche von einem primären Zugrundegehen von Lebergewebe voraus und sieht in der Regeneration und in der Bindegewebsneubildung einen Reparationsvorgang. Obwohl eine ganze Reihe von Tatsachen bekannt sind, die diese Ansichten erschüttern, ich erinnere an den Hinweis von. Jores, daß der Untergang von Lebergewebe, auch wenn er chronisch auftritt, keineswegs von den von Kretz angenommenen Reparationsvorgängen gefolgt sein muß, so sind wir doch nicht in der Lage, die Kretzsche Definition umzuwerfen. Für die vollentwickelte Zirrhose müssen wir sie unter allen Umständen gelten lassen. Die vollentwickelte Zirrhose ist eben ein wohldefiniertes und wohlumschriebenes Krankheitsbild, das wir wohl am ehesten mit der Atherosklerose vergleichen können, insoferne deren Anfangsstadien bis zu einem gewissen Grade umstritten sind, während uns die vollentwickelte Krankheit als ein leicht bestimmbares Bild vor Augen tritt. 
Halten wir uns also streng an die K retzsche Definition, so haben wir manchmal noch die Erscheinung einer anderweit bestimmt charakterisierten Erkrankung der Leber. Hier nehmen wir Tuberkel der verschiedensten Größe oder vielleicht deren Residuen wahr und außerdem eine mehr oder weniger starke bindegewebige Reaktion. Solange sich diese Reaktion im Rahmen eines Ausheilungsvorgangs um die einzelnen Knötchen hält, kann man natürlich nur von einer Lebertuberkulose sprechen. Geht sie aber darüber hinaus und findet sich wohl auch an Stellen, wo keine deutliche Tuberkelbildung nachweisbar oder nicht mehr nachweisbar ist, so ist dieser Erkrankung der Name ,,tuberkulöse Leberzirrhose" zu geben.

Mit dieser Umgrenzung möchte ich hauptsächlich einen Boden schaffen, auf dem weiter gearbeitet werden kann, sie stellt eine reine Beschreibung des Befundes dar, soll aber in keiner Weise über die Ausgänge der Erkrankung Auskunft geben und ebensowenig damit als alleinigen ätiologischen Faktoren den Tuberkelbazillus bezeichnen. Gerade die Frage, ob der Tuberkelbazillus die einzige Ursache einer tuberkulösen Zirrhose sein kann, gehört zu den allerumstrittensten.

Es ist allerdings auch unser Fall nicht geeignet, hier eine Entscheidung zu treffen, weil der stets als Hauptbedingung für das Zustandekommen einer Leberzirrhose bezeichnete Alkohol eine große Rolle gespielt haben kann, da der Patient Weinwirt war. Wir haben hier drei Möglichkeiten:

1. In der zirrhotischen Leber entwickelte sich eine großknotige Tuberkulose.

2. Der Tuberkelbazillus entfaltete gleichzeitig mit dem Alkohol seine zirrhosierende Wirksamkeit.

3. Die Tuberkulose hat allein die Ausbildung der Bindegewebsmassen bewirkt.

Von diesen Möglichkeiten erscheint mir die dritte als die am wenigsten annehmbare. Es scheint mir im Gegensatz zu Kirch nicht wahrscheinlich, daß der Tuberkelbazillus die alleinige Ursache der Erkrankung ist. Wenn man nämlich untersucht, wie sich die Leber gewöhnlich bei einer Infektion mit dem Bazillus verhält, so kann man trotz großer Verschiedenheiten, die wohl von der Menge der Infektionserreger, von ihrer Virulenz und von der Konstitution des Infizierten abhängen, folgende allgemein gültige Gesichtspunkte aufstellen.

Beim frischen Miliartuberkel fehlt jede bindegewebige Reaktion vollkommen. Dauert die Erkrankung etwas länger (z. B. bei einem Fall von 10 Tagen manifester Miliartuberkulose), so kōnnen schon deutliche Bindegewebsumscheidungen der Tuberkel zur Beobachtung kommen. In solchen Fällen kann auch das Glissonsche Gewebe schon etwas verändert sein. Es läßt sich mit der van Giesonfärbung ein Hervortreten des Gewebes nachweisen, das sich am deutlichsten in dem Sichtbarwerden des intralobulären Bindegewebes kund tut, las sonst nur mit Hilfe der Silberimprägnation dargestellt werden kann. Geht der Prozeß weiter, so findet man von den Tuberkeln Bindegewebszüge ausstrahlen, die von Leuko- und Lymphozyten begleitet sind. Bei den Negerm, bei denen manchmal große Teile der Azini durch Tuberkulose zerstört waren, machen sich auch ausnahmslos große Umwälzungen im Lebergewebe geltend. Züge von Bindegewebe gingen inter- und intraazinös von den Tuberkeln ab und stets war eine bedeutende Verdickung der Zentralvenenwand nachweis. 
226 W. Culp: Über großknotige Lebertuberk. kom. m. Leberzirrhose unt. d. Bilde usw.

bar. Niemals aber war es auch nur zu annähernd so mächtigen Bindegewebsbildungen gekommen wie in dem vorliegenden Fall.

$\mathrm{Ob}$ wir nun aber mit der ersten oder zweiten Möglichkeit zu rechnen haben: läßt sich nicht entscheiden. Mir möchte es wohl scheinen, daß die zweite wahrscheinlicher ist, da wir doch auf sehr alte Veränderungen tuberkulöser Natur, ich erinnere an die Perihepatitis und Perisplenitis, in der Oberbauchgegend treffen. Eine Entscheidung ist aber, wie gesagt, hier nieht zu fällen und mit Vermutungen ist keinem gedient. Tch habe mich daher gezwungen gesehen, nur von einer Leberzirrhose allgemein zu sprechen, da eben die Verhältnisse für die Klärung dieser Seite der Frage selten ungünstig liegen.

Beendet im Mai 1920.

\section{Literatur.}

1. Ackermann, Die Histogenese und Histologie der Leberzirrhose. Virchows Arch. $115,1889$.

2. Albrecht, E., Telelogie und Pathologie. Frankf. Zeitschr. f. Pathol. 2, 1. 1908.

3. Askan azy, M., Zur Staubverschleppung und Staubreinigung in den Geweben. Zentralbl. f. Pathol. 17, 642, 1906.

4. Beitzke, H., Über lymphogene retrograde Staubmetastasen. Verhandl. deutsch. pathol. Gesellsch. 1908. XII. 24I.

4a.Derselbe, Diskussion zu Gg. B. Grubers Vortrag auf der 86. Tagung deutscher Naturforscher und Ärzte in Nauheim 1920.

5. Fischer, B., Die Bedeutung der Darminfektion für die Lungentuberkulose. Frankf. Zeitschr. f. Pathol. 5, 1910.

6. Franke, C., Über die Anthrakose retroperitonealer Lymphdrüsen und die Möglichkeit direkter Metastasen von den Brustorganen zu diesen Drüsen. Zieglers Beitr. $54,614.1912$.

7. Gruber, Gg. B., Bemerkungen über Phthise bei Senegalnegern. Zeitsclir. f. Tuberkulose 1920, - „Über Tuberkulose bei Negern, ein Beitrag zur kindlichen Bronchialdrüsenphthise.“ Vortrag tei der 86. Versammlung deutscher Naturforsoher unr Ärzts in Nauheim 1920.

8. Isaak, S., Zur Frage der tuberkulösen Leberzirrhose. Frankf. Zeitschr. f. Pathol. $2,1908$.

9. Jores, L., Zur Kenntnis der subakuten Leberatrophien. Verhandl. deutsch. pathol. Gesellsch. 1907. 320.

10. Kirch, E., Uber tuberkulöse Leberzirrhose, tuberkulöse Schrumpfniere und analoge Folgeerscheinungen granulierender tuberkulöser Entzündung in Pankreas und Mundspeicheldrüsen. Virchows Arch. 225, 129. 1918. Dort weitere Literatur über tuberkulöse Zirrhose.

11. Kretz, Über Leberzirrhose. Wien. klin. Wochenschr. 1900. Heft 12 und Verhandl. deutsch. pathol. Gesellsch. 1904.

12. Kuettner, Die perforierenden Lymphgefäße des Zwerchfells. Beitr. z. klin. Chir. 40, 116. 1903.

13. Lotheissen; G., Über Lebertuberkulose und deren chirurgische Behandlung. Beitr. z. klin. Chir. 81, 1. 1912.

14. Lubarsch, O., Diskussion zum Vortrag von Beitzke. Verhandl. deutsch. pathol. Gesellsch. 1908. XII. 242.

15. Schönberg, S., Leberzirrhose und Tuberkulose. Zieglers Beitr. 59, 601. 1914.

16. Straub, F., Lymphogene Leber- und Milzerkrankungen auf Grund experimenteller Impftuberkulosen. Zeitschr. f. klin. Med. 82, 335. 1916.

17. $S \operatorname{trau} ß, J$, Über die Resorption der Tuberkelbazillen aus dem Darm. Frankf. Zeitschr. f. Pathol. 5, 447. 1910.

18. Tendeloo, N. Ph., Allgemeine Pathologie. Springer, Berlin 1919. 66 u. 416.

19. Ziegler, Kurt, Die Hodgkinsche Krankheit. G. Fischer, Jena 1911. 